\title{
The Quark Model via an hbar expansion of QCD
}

\author{
Paul Hoyer* \\ Department of Physics and Helsinki Institute of Physics \\ POB 64, FIN-00014 University of Helsinki, Finland \\ E-mail: paul.hoyer@helsinki.fi
}

I discuss the possibility that the quark model emerges as the lowest order of an $\hbar$ expansion of QCD bound states. In a hamiltonian approach the instantaneous $A^{0}$ potential is determined by the field equations separately for each Fock component. These equations allow also a linear potential as a homogeneous solution. Stationarity of the action sets the direction of the ensuing constant electric field to be along the fermion pair separation. States bound by this non-perturbative, linear $A^{0}$ potential are analogous to the Born term of standard perturbative expansions of scattering amplitudes, in that they represent the dominant contribution at lowest order in $\hbar$ (no loops) and at $\mathscr{O}(g)$ in the potential.

The Dirac equation for relativistic fermions bound by an external $A^{0}$ potential is most easily derived using retarded boundary conditions. I demonstrate why this boundary condition does not affect the bound state energies at lowest order in $\hbar$. Translated to physical Feynman boundary conditions the Dirac bound states are a superposition of Fock states with any number of fermionantifermion pairs.

Applying this approach to relativistic quark-antiquark states in QCD results in a bound state equation which was previously proposed without derivation and shown to provide a reasonable description of the meson spectrum, including linear Regge trajectories. The equal-time wave functions have unique Lorentz transformation properties, which ensure the correct dependence of the bound state energy on the center-of-mass momentum. This indicates that the solution is exact at the Born level, i.e., at lowest order in $\hbar$ and at $\mathscr{O}(g)$ in the QCD interaction.

European Physical Society Europhysics Conference on High Energy Physics

July 16-22, 2009

Krakow, Poland

\footnotetext{
* Speaker.
} 


\section{Introduction}

The perturbative expansion of scattering amplitudes is a powerful tool. In particular, the amplitudes display the (conserved) symmetries of the lagrangian at each order in the coupling $g$ (and the Planck constant $\hbar$ ). However, the expansion diverges at the locations of bound state poles. For bound states a formally exact approach is provided by the Bethe-Salpeter (BS) framework [1]. The BS equation does not close and hence requires truncations. However, any finite $\mathscr{O}\left(g^{n}\right)$ perturbative approximation of the BS kernel violates the boost invariance of bound states, as seen in the derivation of the Dirac equation from the BS equation [2].

It thus appears that a general principle is needed to separate essentially "kinematic" contributions (e.g., those required by Lorentz invariance) from "dynamic" higher order corrections. This may be provided by an expansion in the Planck constant $\hbar$. From the path integral representation of field theory it is clear that the $\hbar$ and loop expansions are equivalent. In Feynman diagrams the power of the coupling $g$ is strictly correlated to the number of loops. Bound state wave functions on the other hand contain all powers of $g$, yet may be of lowest order in the loop expansion. This is again illustrated by the Dirac equation, which is obtained by iterating scattering from a fixed classical potential, without including loop corrections on the propagators or vertices.

In the $\hbar \rightarrow 0$ limit the phase $\exp (i S / \hbar)$ in the path integral oscillates rapidly, causing fields to approach their classical values (corresponding to a stationary action $S$ ). The dominant binding is provided by the instantaneous $A^{0}$ gauge field which is a solution of the equations of motion and thus depends on the positions of the charged particles in each Fock component of the bound state. The instantaneity of $A^{0}$ is due to the absence of its time derivative in the lagrangian. Gauges where no $\partial_{0} A^{0}$ term is introduced through gauge fixing are therefore preferred (i.e., Coulomb gauge $\vec{\nabla} \cdot A=0$ rather than Feynman gauge $\partial_{\mu} A^{\mu}=0$ ).

In the following I sketch ${ }^{1}$ a hamiltonian approach to fermion-antifermion bound states which appears to be exact at lowest order in $\hbar$ and in the gauge coupling $g$. It provides the equivalent of a "Born term" for gauge theory bound states, to be systematically improved through higher order corrections in $\hbar$ and $g$. The wave functions in different reference frames are related, as required for studies of bound state scattering.

\section{The linear potential option}

The Schrödinger equation for ordinary QED atoms (muonium, $e^{-} \mu^{+}$) is readily derived in a hamiltonian approach to field theory in the semi-classical limit, i.e., at lowest order in $\hbar$ (no loops). The instantaneous $A^{0}$ potential determined by the equations of motion is specific to each Fock state component. This demonstrates the distinction between the $A^{0}$ field for a given Fock state and the $A^{0}$ field that would be measured by an external probe, the latter being given by a superposition of all contributing Fock states.

The determination of $A^{0}$ from the QED field equation for the Fock component where the electron is located at $\vec{x}_{1}$ and the muon at $\vec{x}_{2}$,

$$
-\vec{\nabla}_{\vec{x}}^{2} A^{0}\left(\vec{x} ; \vec{x}_{1}, \vec{x}_{2}\right)=e \delta^{3}\left(\vec{x}-\vec{x}_{1}\right)-e \delta^{3}\left(\vec{x}-\vec{x}_{2}\right)
$$

\footnotetext{
${ }^{1}$ A more complete account is given in arXiv:0909.3045.
} 
is unique only up to a homogenous solution. In particular, a contribution linear in $\vec{x}$ may be added,

$$
A^{0}\left(\vec{x} ; \vec{x}_{1}, \vec{x}_{2}\right)=\Lambda^{2} \hat{\ell} \cdot \vec{x}+\frac{e}{4 \pi}\left(\frac{1}{\left|\vec{x}-\vec{x}_{1}\right|}-\frac{1}{\left|\vec{x}-\vec{x}_{2}\right|}\right)
$$

where the magnitude $\Lambda$ and the direction $\hat{\ell}$ of the linear term are free parameters. The action contains a finite interference term between the homogeneous and Coulomb terms ${ }^{2}$,

$$
-\frac{1}{4} \int d^{3} \vec{x} F_{\mu v} F^{\mu v}=\frac{1}{2} \Lambda^{4} \int d^{3} \vec{x}+\frac{1}{3} e \Lambda^{2} \hat{\ell} \cdot\left(\vec{x}_{1}-\vec{x}_{2}\right)-\frac{e^{2}}{4 \pi} \frac{1}{\left|\vec{x}_{1}-\vec{x}_{2}\right|}
$$

Stationarity of the action wrt. variations in the direction of the linear potential requires $\hat{\ell}$ to be parallel to $\vec{x}_{1}-\vec{x}_{2}$. Hence (choosing the sign to give an attractive potential)

$$
A^{0}\left(\vec{x} ; \vec{x}_{1}, \vec{x}_{2}\right)=\Lambda^{2} \frac{\vec{x}_{1}-\vec{x}_{2}}{\left|\vec{x}_{1}-\vec{x}_{2}\right|} \cdot \vec{x}+\frac{e}{4 \pi}\left(\frac{1}{\left|\vec{x}-\vec{x}_{1}\right|}-\frac{1}{\left|\vec{x}-\vec{x}_{2}\right|}\right)
$$

$\Lambda$ must be a universal constant, $\Lambda \neq \Lambda\left(\vec{x}_{1}, \vec{x}_{2}\right)$, for the $\mathscr{O}\left(\Lambda^{4}\right)$ term in (2.3) not to introduce an infinite relative phase between Fock states. The linear potential appears to be consistent with all requirements and allows to incorporate non-perturbative features of the theory at the semi-classical level. The interaction energy of the fermions with the $A^{0}$ field is then,

$$
e\left[A^{0}\left(\vec{x}_{1} ; \vec{x}_{1}, \vec{x}_{2}\right)-A^{0}\left(\vec{x}_{2} ; \vec{x}_{1}, \vec{x}_{2}\right)\right]=e \Lambda^{2}\left|\vec{x}_{1}-\vec{x}_{2}\right|-2 \frac{e^{2}}{4 \pi} \frac{1}{\left|\vec{x}_{1}-\vec{x}_{2}\right|}
$$

Since both the electron and the muon interact the Coulomb potential is counted twice. However, according to (2.3) the phase originating from the gauge part of the action $\exp \left(-\frac{i}{4} \int d t \int d^{3} \vec{x} F_{\mu \nu} F^{\mu v}\right)$ reduces the Coulomb potential to its physical value, $V_{C}=-\alpha / r$.

The experimental fact that QED does not confine electric charge compels us to choose $\Lambda_{Q E D}=$ 0 and thus recover the standard description of muonium bound only by the Coulomb potential. However, there is a corresponding linear potential solution also of the QCD equations of motion. Choosing $\Lambda_{Q C D} \neq 0$ amounts to a novel boundary condition for perturbation theory, motivated by the non-trivial structure of the true vacuum of QCD.

A linear potential extending to infinity would be unacceptable on physical grounds - not only quarks but also gluons are confined. However, $A^{0}\left(\vec{x} ; \vec{x}_{1}, \vec{x}_{2}\right)$ of (2.4) is the field of only a single Fock state. The field that would be measured by an external probe is a superposition of the contributions from all Fock components. As seen from (2.4), Fock components with opposite separations $\vec{x}_{1}-\vec{x}_{2}$ give opposite contributions to $A^{0}$ which cancel if the corresponding Fock probabilities are the same. Hence the electric field vanishes outside the bound state, where all Fock states contribute coherently.

The linear potential contributes at $\mathscr{O}(e)$ since $\Lambda$ is non-perturbative, see (2.5). Hence in a perturbative sense it dominates the single (Coulomb and transverse) photon exchanges which are of $\mathscr{O}\left(e^{2}\right)$. This allows the analog of a "Born term" for gauge theory bound states, which is exact at lowest order in $\hbar$ (no loops) and at $\mathscr{O}(g)$ in the coupling. The Lorentz invariance of the theory

\footnotetext{
${ }^{2}$ The infinite self-energy contribution of the Coulomb potential may be neglected since it is the same for all Fock states.
} 
must be intact at each order of $\hbar$ and $g$. In particular, the bound state energy $E$ should have the correct dependence, $E=\sqrt{M^{2}+\vec{k}^{2}}$, on the CM momentum $\vec{k}$. The bound state equation given here for wave functions at equal time in all frames in fact has this non-trivial and unique property. In QED atoms without a linear potential, on the other hand, the $A^{0}$ Coulomb potential dominates transverse photon exchange only in the rest frame. The boosted muonium atom gets a leading $\mathscr{O}\left(e^{2}\right)$ contribution from $|e \mu \gamma\rangle$ Fock states with a transverse photon which must be included to get the correct $\vec{k}$ dependence [3].

\section{The Dirac equation - retarded boundary conditions}

Particle production cannot be neglected when the dynamics is relativistic. An $|e \mu\rangle$ state at $t=0$ will with time develop into states with additional electron and muon pairs if the interaction potential $A^{0}$ is commensurate with the particle masses. Relativistic bound states (defined at equal time of the constituents) therefore have Fock states with arbitrarily many particles.

The Dirac wave function $\psi(\vec{x})$ of an electron bound in an external potential is relativistic yet describes the spatial distribution of a single (positive or negative energy) electron. Understanding the apparent absence of multi-particle Fock states in the Dirac wave function turns out to be useful for formulating a relativistic hamiltonian description of $e^{-} \mu^{+}$atoms and hadrons.

The Green function $G\left(p^{0}, \vec{p}\right)$ of an electron in a static (time-independent) $A^{0}$ potential (Fig. 1) satisfies

$$
G\left(p^{0}, \vec{p}\right)=S+S K G
$$

where $S$ is the electron propagator and $K$ the kernel for a single $A^{0}$ interaction. The $p^{0}$ component of

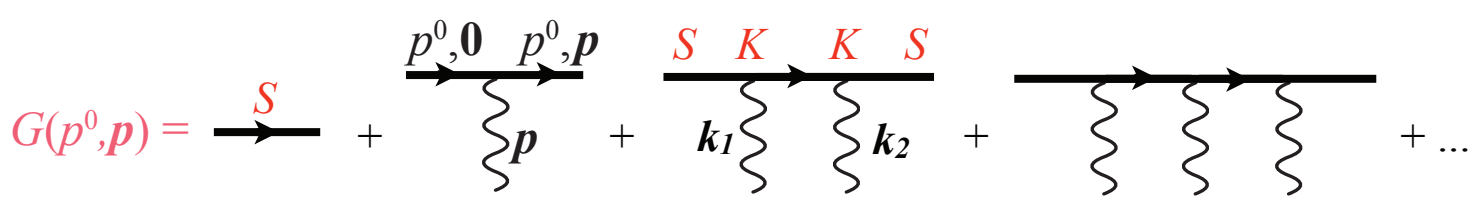

Figure 1: Electron scattering from a static external potential. The energy $p^{0}$ of the electron does not change during the scattering. The initial and final electron momenta are denoted $\left(p^{0}, \overrightarrow{0}\right)$ and $p=\left(p^{0}, \vec{p}\right)$.

the electron's 4-momentum is conserved during the scattering since the static source only transfers 3-momentum. The initial and final 3-momenta of the electron are taken to be $\overrightarrow{0}$ and $\vec{p}$, respectively.

The Green function has poles at the bound state energies $E_{R}$,

$$
G\left(p^{0}, \vec{p}\right)=\frac{R\left(E_{R}, \vec{p}\right)}{p^{0}-E_{R}}+\ldots
$$

The pole residue $R\left(E_{R}, \vec{p}\right)$ satisfies $R=S K R$, or explicitly (with $p^{0}=E_{R}$ )

$$
R\left(E_{R}, \vec{p}\right)=\frac{i}{\not p-m+i \varepsilon} \int \frac{d^{3} \vec{k}}{(2 \pi)^{3}}(-i e) \gamma^{0} A^{0}(\vec{k}) R\left(E_{R}, \vec{p}-\vec{k}\right)
$$

Multiplying by $\not p-m$ and Fourier transforming to $\left(p^{0}, \vec{x}\right)$-space we find that the residue $R$ satisfies the Dirac equation,

$$
\left[-i \vec{\nabla} \cdot \vec{\gamma}+e \gamma^{0} A^{0}(\vec{x})+m\right] R\left(E_{R}, \vec{x}\right)=E_{R} \gamma^{0} R\left(E_{R}, \vec{x}\right)
$$


In order to display the equal-time Fock states of the bound state given by the Dirac equation we need to time-order the interactions. At $\mathscr{O}\left(e^{2}\right)$ the first (second) diagram on the rhs. of Fig. 2 corresponds to the intermediate electron having positive (negative) energy, $E_{i}= \pm \sqrt{\vec{k}_{i}^{2}+m_{e}^{2}}$. According

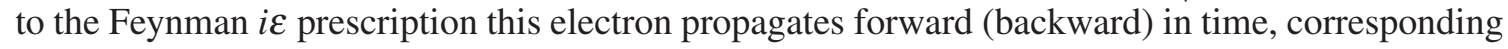
to an intermediate $\left|e^{-}\right\rangle\left(\left|e^{-} e^{+} e^{-}\right\rangle\right)$Fock state. At higher orders in $e$ further time orderings contribute. Consequently the bound state has Fock components with arbitrarily many $e^{+} e^{-}$pairs. The creation and destruction of the various Fock components balance to create a bound state which is stationary in time.
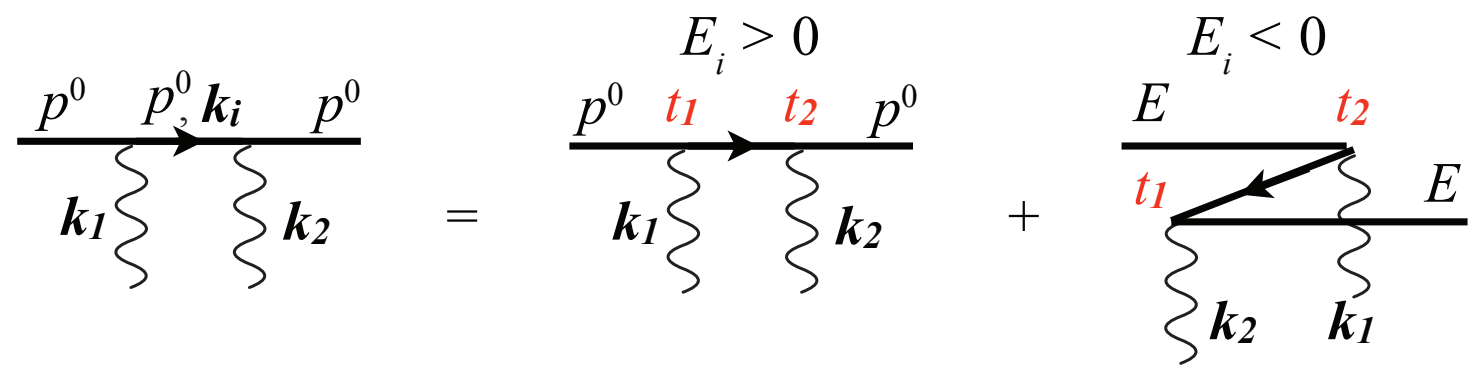

Figure 2: Electron scattering from a static $A^{0}$ potential at $\mathscr{O}\left(e^{2}\right)$. The covariant $\left(p^{0}\right.$-conserving) diagram on the $l h s$. splits under time-ordering into the two diagrams on the rhs. The intermediate electron has 3momentum $\vec{k}_{i}$ and energy $E_{i}= \pm \sqrt{\vec{k}_{i}^{2}+m_{e}^{2}}$. The negative energy electron propagates backward in time, implying the creation (annihilation) of an $e^{+} e^{-}$pair at $t_{1}\left(t_{2}\right)$.

The Dirac wave function $\psi(\vec{x})$ describes only a single electron, not the details of the multiparticle bound state dynamics. As seen above, the bound state energies $p^{0}=E_{R}$ given by the poles of the Green function (3.2) nevertheless are eigenvalues of the (time independent) Dirac equation (3.4) satisfied by the pole residues $R\left(E_{R}, \vec{x}\right)$. This single particle Dirac wave function may be given the following hamiltonian interpretation.

The static potential conserves the energy component $p^{0}>0$ of the electron momentum. Hence the covariant Feynman diagrams in Fig. 1 which build the Green function $G\left(p^{0}, \vec{p}\right)$ do not depend on the Feynman $i \varepsilon$ prescription at the negative energy pole of the electron propagator, $p^{0}=$ $-\sqrt{\vec{k}_{i}^{2}+m_{e}^{2}}+i \varepsilon$. In particular, the bound state energies $E_{R}$ of the Green function (3.2) will not change if instead of the Feynman propagator of the electron we use the retarded one,

$$
S_{R}\left(p^{0}, \vec{p}\right)=i \frac{\not p+m_{e}}{\left(p^{0}-E_{p}+i \varepsilon\right)\left(p^{0}+E_{p}+i \varepsilon\right)}
$$

where $E_{p}=\sqrt{\vec{p}^{2}+m_{e}^{2}}$. However, this changes the time development of the scattering so that the electron always propagates forward in time,

$$
S_{R}(t, \vec{p})=\frac{\theta(t)}{2 E_{p}}\left[\left(E_{p} \gamma^{0}-\vec{p} \cdot \vec{\gamma}+m_{e}\right) e^{-i E_{p} t}+\left(E_{p} \gamma^{0}+\vec{p} \cdot \vec{\gamma}-m_{e}\right) e^{i E_{p} t}\right]
$$

Consequently the second (Z-)diagram on the rhs. of Fig. 2 is absent, while the first diagram refers to a positive or negative energy electron moving forward in time. There are only one-electron Fock 
states and the Dirac wave function describes the distribution of this electron. This single particle picture of the time development obtained using retarded boundary conditions does not correspond to the physical Fock state structure. Furthermore, the argument that the bound state energies $\left(E_{R}\right.$ in (3.2)) are independent of the boundary condition only holds in the absence of loops, i.e., in the $\hbar \rightarrow 0$ limit. In fact, loops evaluated with retarded propagators vanish since the poles are all on the same side of the real axis.

In the absence of backward propagation the electron becomes localized in space as the propagation time vanishes,

$$
\lim _{t \rightarrow 0^{+}} S_{R}(t, \vec{x})=\gamma^{0} \delta^{3}(\vec{x})
$$

which makes a hamiltonian approach feasible.

In the operator formalism the retarded boundary condition is implemented using the "retarded vacuum",

$$
|0\rangle_{R}=N^{-1} \prod_{\vec{p}, \lambda} d_{\vec{p}, \lambda}^{\dagger}|0\rangle
$$

where the product is over all momenta $\vec{p}$ and helicities $\lambda$. The normalization factor $N$ is fixed by ${ }_{R}\langle 0 \mid 0\rangle_{R}=1$. The retarded vacuum satisfies

$$
b_{\vec{p}, \lambda}|0\rangle_{R}=d_{\vec{p}, \lambda}^{\dagger}|0\rangle_{R}=0 \quad \text { and hence } \quad \psi(x)|0\rangle_{R}=0
$$

where $\psi(x)$ is the free (interaction picture) fermion field. Consequently the retarded propagator (3.6) is given by the standard operator matrix element in the retarded vacuum,

$$
S_{R}(x-y)={ }_{R}\langle 0|T[\psi(x) \bar{\psi}(y)]| 0\rangle_{R}
$$

The negative energy contribution to the propagator arises from the $d^{\dagger} d$ term, which represents the removal of a positive energy antifermion from $|0\rangle_{R}$. The interaction hamiltonian annihilates the retarded vacuum,

$$
H_{I}(t)|0\rangle_{R}=e \int d^{3} \vec{x} A^{0}(\vec{x}) \psi^{\dagger}(t, \vec{x}) \psi(t, \vec{x})|0\rangle_{R}=0
$$

which ensures the absence of particle production. The Dirac equation may be readily derived using this hamiltonian approach with retarded boundary conditions.

\section{Relativistic $f \bar{f}$ bound states}

The instantaneity of the $A^{0}$ potential is a consequence of the absence of a $\partial_{0} A^{0}$ term in the lagrangian, and does not require non-relativistic motion of the sources. The dominance of $A^{0}$ over the transverse $\vec{A}$ gauge field components in a perturbative expansion holds for non-relativistic motion (atoms in their rest frame) and more generally with a non-perturbative linear $A^{0}$ potential as in (2.5). At lowest order in $\hbar$ the use of the retarded vacuum (3.8) as boundary condition sums implicitly over fermion pair production by the $A^{0}$ potential, as discussed above for the Dirac equation.

An equal-time $f \bar{f}$ bound state equation is obtained with a hamiltonian approach in the retarded vacuum, using the linear part of the $A^{0}$ potential (2.4). The equation is expected to be exact at leading orders in $\hbar$ and the coupling $g$, giving the equivalent of a Born term for bound states. For QCD mesons there is a solution where the linear potential is present only in the center elements 
$(a=3,8)$ of the $\mathrm{SU}_{3}$ color group. The $q \bar{q}$ color singlet wave function $\chi_{\vec{k}}^{\alpha \beta}(\vec{x})$, where $\vec{k}$ is the $\mathrm{CM}$ momentum of the bound state, $\alpha$ and $\beta$ are Dirac indices and $\vec{x}$ is the spatial separation of the quark pair at an instant of time, satisfies the bound state equation

$$
-i \vec{\nabla} \cdot\left[\alpha, \chi_{\vec{k}}(\vec{x})\right]+\frac{1}{2} \vec{k} \cdot\left\{\alpha, \chi_{\vec{k}}(\vec{x})\right\}+m_{1} \gamma^{0} \chi_{\vec{k}}(\vec{x})-\chi_{\vec{k}}(\vec{x}) \gamma^{0} m_{2}=(E-V) \chi_{\vec{k}}(\vec{x})
$$

Here $m_{1}\left(m_{2}\right)$ is the mass of the $f(\bar{f})$ constituent, $\alpha=\gamma^{0} \vec{\gamma}$ is a standard Dirac matrix and $V(\vec{x})=$ $g \Lambda^{2}|\vec{x}|$ is the linear potential. This equation is similar in form to the one proposed by Breit [4] already in 1929. Our derivation is valid at leading order in the gauge coupling $g$, consequently the potential is purely linear. The properties of this equation (with $\vec{k}=0$ and $m_{1}=m_{2}$ ) was studied phenomenologically [5] using a linear + Coulomb potential (see also [6] and references therein). It was previously seen to follow from stationary phase arguments assuming retarded boundary conditions [7].

Due to the underlying gauge invariance it is not surprising that the energy $E$ of the bound state appears in the canonical form $E-e A^{0}$ in (4.1), while the CM momentum $\vec{k}$ is kinematic since $\vec{A}=0$. After a separation of the angular dependence in the rest frame (with $m_{1}=m_{2}$ ) the radial components $F_{i}(r)$ of the wave function were found [5] to be potentially singular at $r=0$ and at $E-V(r)=0$. Requiring local normalizability at these points resulted in quantized energy levels and a reasonable spectrum, including linear Regge trajectories. The bound state equation reduces as expected to the Schrödinger equation in the non-relativistic limit $(V \ll E)$, in which case the singular point $E-V(r)=0$ moves to $r=\infty$.

In the relativistic case the linear potential $V(r)$ dominates on the rhs. of (4.1) at large distances $r$ between the quarks, and can be balanced only by a large derivative term on the lhs. The wave function is then rapidly oscillating $\sim \exp \left(i g \Lambda^{2} r^{2} / 4\right)$ and has an $r$-independent probability density. This wave function, which is obtained with retarded boundary conditions, is (as in the Dirac case above) not directly related to the physical Fock state structure. A multi-particle bound state is normalized by squaring the wave function of each Fock component, integrating over its constituent d.o.f.'s and finally summing over the various Fock state probabilities. In a linear potential the energy density, and plausibly also the number of "sea" quarks polarized from the vacuum, increases linearly with the distance between the valence quarks. The (divergent) normalization $\propto r$ of the wave function (4.1) obtained using retarded boundary conditions may reflect these virtual sea quarks. This question requires more study, also for $\vec{k} \neq 0$ and $m_{1} \neq m_{2}$.

Since the wave function $\chi_{\vec{k}}(\vec{x})$ describes constituents at equal time for all CM momenta $\vec{k}$ it is not explicitly Lorentz covariant. Nevertheless, if the bound state equation (4.1) is accurate to lowest order in $\hbar$ and $g$ the bound state energy must have the correct dependence on the CM momentum, $E(\vec{k})=\sqrt{\vec{k}^{2}+M^{2}}$. Remarkably, this turns out to be the case [8]. The $\vec{k}$-dependence of the wave function is explicit in $1+1$ dimensions. In $3+1$ dimensions $\chi_{\vec{k}}(\vec{x})$ with $\vec{x} \| \vec{k}$ can be expressed in terms of the $\vec{k}=0$ wave function. This also holds for the first derivative of $\chi_{\vec{k}}$ wrt. $\vec{x} \perp \vec{k}$, which allows to (numerically) determine $\chi_{\vec{k}}(\vec{x})$ for all $\vec{x}$ based on the $\vec{k}=0$ solution.

The $\vec{k}$-dependence of the wave function $\chi_{\vec{k}}$ and of its energy eigenvalues $E(\vec{k})$ are found as follows [8]. For $\vec{k}=(0,0, k)$ along the $z$-axis, the bound state equation (4.1) for $\chi_{\vec{k}}(0,0, z)$ and its first derivatives $\partial_{i} \chi_{\vec{k}}(0,0, z),(i=x, y)$ becomes independent of $k$ when the coordinate $z$ is expressed 
in terms of the variable $s$ defined by

$$
d z=\frac{2}{E(k)-V(z)} d s
$$

In the non-relativistic regime, $V \ll E$, this is just the Lorentz contraction expected for distances measured at equal time in every frame. The fact that the transformation is governed by the canonical energy $E-V(z)$ means that the contraction rate for a relativistic wave function is $z$-dependent. The Lorentz covariance of (4.1) requires furthermore [8] that the square of the canonical fourmomentum $P=(E-V, 0,0, k)$ is frame independent when expressed as a function of $s$. For a linear potential $V(z)=g \Lambda^{2}|z|$ the relation (4.2) integrates (taking $z>0$ ) to

$$
s=\frac{1}{2} z\left(E-\frac{1}{2} V\right)
$$

With $E^{2}-k^{2}=M^{2}$ we then have indeed that

$$
P^{2}=(E-V)^{2}-k^{2}=M^{2}-4 g \Lambda^{2} s
$$

is independent of $k$. This seeming coincidence only holds for a linear potential.

\section{Concluding remarks}

This study was motivated by the success of the quark model, which suggests that a semiclassical treatment of hadrons can give a reasonable first approximation. The study of field theory bound states is complicated by the fact that usual perturbative expansion diverges at the bound state energies. An infinite number of Feynman diagrams need to be summed in order to generate bound states. A hint as to a possible approach is that the ladder (loop) diagrams describing the scattering of a light particle from a heavy partner generate the Dirac equation in the limit when the heavy particle mass tends to infinity [2]. The Dirac equation describes scattering from the classical Coulomb field of the heavy source. Thus the divergence of the series is not generated by true loop contributions, but by the dominance of scattering from a classical field, which is of lowest order in $\hbar$.

The $\hbar \rightarrow 0$ approximation preserves the symmetries of the theory (such as gauge and Lorentz invariance), and is applicable in any dynamics. It allows to relate bound states in different frames and should thus enable, e.g., the study of bound state scattering. The absence of loops allows to use retarded boundary conditions which implicitly sums over all particle production. Since loop integrals vanish for retarded propagators (all poles being in the same hemisphere of the complex plane) the use of retarded propagators ensures that only effects of lowest order in $\hbar$ are included. It is obviously important to demonstrate how loop corrections to the bound states discussed here can be systematically calculated.

A central issue which remains to be studied is how to take into account the effects of spontaneous chiral symmetry breaking in QCD. This requires the use of a ground state which is not chirally symmetric. 


\section{Acknowledgments}

I wish to thank the organizers of the EPS-HEP2009 parallel session on hadron physics for their invitation to give this contribution, as well as the CERN theory division for their hospitality. I am grateful for travel support from the Magnus Ehrnrooth foundation. Special thanks are due to Stan Brodsky for his advice on bound state structure and inspiring discussions. I have also benefitted from discussing with Dennis Dietrich, Stan Glazek, Matti Järvinen, Stéphane Peigné, Jian-Wei Qiu, Patta Yogendran and Feng Yuan.

\section{References}

[1] E. E. Salpeter and H. A. Bethe, Phys. Rev. 84 (1951) 1232;

G. P. Lepage, SLAC-R-212 (1978)

http://www.slac.stanford.edu/cgi-wrap/getdoc/slac-r-212.pdf

[2] D. R. Yennie, Brandeis Summer Institute Lectures on Strong and Electromagnetic Interactions (Brandeis University, Waltham, Mass., 1963), Vol. I.;

S. J. Brodsky, In *Brandeis Univ 1969, Proceedings, Atomic Physics and Astrophysics, Vol.1*, New York 1971, 91-169; and SLAC-PUB-1010 (1971);

E. Brezin, Thesis Université de Paris. Paris, France 1970;

W. Dittrich, Phys. Rev. D 1 (1970) 3345;

A. R. Neghabian and W. Glöckle, Can. J. Phys. 61 (1983) 85.

[3] M. Järvinen, Phys. Rev. D 71 (2005) 085006 [arXiv:hep-ph/0411208].

[4] G. Breit, Phys. Rev. 34 (1929) 553 and

[5] D. A. Geffen and H. Suura, Phys. Rev. D 16 (1977) 3305.

[6] W. Krolikowski, Acta Phys. Polon. B 23 (1992) 43.

[7] P. Hoyer, Phys. Scripta 30 (1984) 29, Phys. Lett. B 145 (1984) 127 and Phys. Lett. B 148 (1984) 199.

[8] P. Hoyer, preprint HU-TFT-85-49 (http://www-lib.kek.jp/cgi-bin/img_index?8601069) and Phys. Lett. B 172 (1986) 101. 